\title{
REMARQUES SUR LA THÉORIE CAPILLAIRE DE LAPLAGE;
}

Par G. Vax der MENSBRUGGHE.

Dans un article intitulé Tension superficielle, et inséré au Journal de Physique (1), je lis les lignes suivantes, qui servent d'entrée en matière : “Pour expliquer les phénomènes capillaires, on admet que chaque molécule est attirée par toute molécule situèe à une distance plus pelite que le rayon d'activité moléculaire; on démontre alors que la surface libre supporte en chaque point une pression normale dont la valeur est donnée par la formule de Laplace

$$
p=\mathbf{K}+\mathbf{H}\left(\frac{\mathbf{I}}{\mathbf{R}}+\frac{\mathbf{r}}{\mathbf{R}^{\prime}}\right),
$$

dans laquelle $\mathbf{R}$ et $R^{\prime}$ désignent les rayons de courbure principaux au point considéré; ces rayons sont comptés posilivement quand la surface est convexe. Le terme $\mathrm{K}$ a pour effet d'augmenter la pression hydrostatique exercée par le milieu ambiant en chaque point de la surface du liquide; on peut donc raisonner comme si la pression moléculaire ne comportait qu'un seul terme

$$
\mathrm{H}\left(\frac{\mathbf{I}}{\mathbf{R}}+\frac{\mathbf{I}}{\mathbf{R}^{\prime}}\right) \cdot \cdots
$$

En rappelant ainsi la célèbre théorie de Laplace, l'auteur de l'article, M. Chervet, semble croire qu'elle est de tout point conforme à l'observation directe; or il n'en est rien, comme je l'ai fait voir. dans une Note présentée en 1886 au Congrès de l'Association frauȩaise pour l'avancement des Sciences (Congrès de Nancy). Qu'il me soit permis de rappeler brièvement quelques-unes des considérations que j'ai fait valoir à celte époque.

Et tout d'abord, le terme $K$ représente-t-il une pression susceptible d'être transmise partout au sein de la masse liquide? Pour le savoir, il suffit de se rappeler que, d'après Laplace même, K doit diminuer en général quand la température du liquide s'élève, et de faire l'expérience suivante : dans un vase cylindrique de $o^{\mathrm{m}}, 30$

(') Joir t. VII, p. '485; octobre I $\$ 88$. 
à $0^{\mathrm{m}}, 40$ de diamètre, on verse de l'eau pure à $\mathrm{I} \mathrm{5}^{\circ} \mathrm{C}$., jusqu'à ce qu'elle déborde; quand la masse est en repos, on approche d'un point quelconque $a$ de la surface libre une tige métallique chauffée au rouge; la chaleur rayonnée vers la portion liquide sous-jacente élève la température de celle-ci, et dès lors la pression moléculaire qui correspond à la température croissante doit aller en diminuant; on s'attend donc à voir le niveau du liquide s'élever en $a$; mais c'est précisépent l'inverse qui a lieu : l'eau qui environne $a$ fuit de toutes parts, comme si elle obéissait à une force centrifuge émanée de ce même point, et la surface liquide y devient légèrement creuse. Conclusion: le terme K n'exprime pas une pression susceptible d'ètre accusée par l'expérience.

Quant au terme $H\left(\frac{I}{R}-\frac{I}{R^{\prime}}\right)$, il représente réellement une pression dont l'existence a été démontrée par des preuves innombrables. Mais il faut remarquer que Laplace obtient le terme en question en calculant l'effet d'un ménisque compris entre la surface libre, le plan tangent au point considéré et un hémisphère ayant pour diamètre le double rayon $r$ d'activité de l'attraction moléculaire; or on sait aujourd'hui que le rayon $r$ est inférieur à $\frac{1}{20000}$ de millimètre; d'autre part, on peut concevoir $R$ et $R^{\prime}$ suffisamment grands pour que le ménisque ci-dessus s'évanouisse : dès lors

$$
\mathbf{H}\left(\frac{\mathrm{I}}{\mathbf{R}}+\frac{\mathrm{I}}{\mathrm{R}^{\prime}}\right)
$$

s'annulerait et la théorie de Laplace conduirait à la conséquence que la pression due à une surface sphérique, par exemple, peut parfois être nulle, ce qui serait en contradiction formelle avec l'expérience directe; car, on le sait, une bulle de savon de $\mathrm{o}^{\mathrm{n}}, 20$ de diamètre, attachée au bord d'un entonnoir dont le bec est ouvert, chasse l'air qui a servi à la gonfler, ce qui n'arriverait pas si les deux pressions normales, dues aux deux faces de la lame, étaient nulles. Et pourtant comment concevoir encore le ménisque en question dans le cas où $R$ et $\mathrm{R}^{\prime}$ valent $\mathrm{o}^{\mathrm{m}}$, $1 \mathrm{o}$, c'est-à-dire deux millions de fois le rayon $r$ de l'attraction moléculaire? Cette objection a été présentée pour la première fois en i 880 par M. Marangoni, de Florence.

On le voit, la théorie de Laplace conduit à des conséquences 
absolument contraires aux résultats de l'observation; j’ajouterai qu'il est impossible de concilier l'existence d'une pression normale K (très considérable d'après l'illustre auteur lui-même), dirigée vers l'intérieur du liquide, avec l'évaporation qui a lieu continuellement à la surface. Il ne faut pas s'étonner d'un pareil désaccord, car Laplace a raisonné sur un liquide incompressible et soumis exclusivement à des forces attractives, par conséquent sur un liquide idéal dont les propriétés sont tout à fait différentes de celles des liquides naturels; ceux-ci sont compressibles, et leurs molécules sont sollicitées non seulement par des forces at tractives, mais encore par des forces répulsives dont l'intensité croît ou décroît, suivant la distance, plus rapidement que les premières forces; c'est grâce à ces forces répulsives que, contrairement à ce qu'on a enseigné depuis si longtemps, la couche superficielle d'un liquide n'est jamais en équilibre dans les conditions ordinairement supposées; c'est encore grâce à elles, combinées avec les forces attractives, qu'il se produit précisément une force contractive dans cette couche, ainsi que je me suis efforcé de l'établir dans mes études Sur l'instabilité de l'équilibre de la couche superficielle d'un liquide ( $\left.{ }^{1}\right)$.

Je ne puis donc nullement me rallier à l'opinion de M. Chervet, d'après laquelle il conviendrait de présenter la tension superficielle comme une conséquence de la pression moléculaire; selon moi, c'est précisément l'inverse qu'il convient de faire : la dernière méthode présente l'avantage précieux de ne conduire qu'à des conséquences toujours conformes à l'expérience; c'est là, il faut bien l'avouer, un avantage que les calculs les plus profonds, appliqués à l'hypothèse de substances purement idéales, ne sauraient remplacer. A cet égard, la théorie capillaire de Laplace devra toujours exciter l'admiration de tous les analystes, mais les physiciens ne pourront en tirer aucun parti, précisément parce qu'elle n'est applicable qu'à des substances hypothétiques et essentiellement différentes des liquides naturels.

(') Bulletin de l'Acad. roy. de Belgique, t. XI, p. 341, et t. XII, p. 623 ; 8886 . 